\title{
REVISIÓN DEL PUNTO DE CORTE ENTRE LOS ESTADIOS T1 Y T2 EN LA CLASIFICACIÓN TNM DEL CARCINOMA RENAL DE 1997
}

\author{
A.J. LÓPEZ LUQUE, F.J. ANGLADA CURADO, J.L. CARAZO CARAZO, \\ J. ÁLVAREZ KINDELAN, J. MÁRQUEZ LÓPEZ, M. LEVA VALLEJO, \\ J.C. REGUEIRO LÓPEZ, R. PRIETO CASTRO, M.J. REQUENA TAPIA
}

Servicio de Urología. Hospital Regional Universitario Reina Sofía. Córdoba.

Actas Urol Esp. 27 (4): 292-296, 2003

\section{RESUMEN}

"REVISIÓN DEL PUNTO DE CORTE ENTRE LOS ESTADIOS T1 Y T2 EN LA CLASIFICACIÓN TNM DEL CARCINOMA RENAL DE 1997"

OBJETIVO: Realizamos un estudio retrospectivo de los pacientes tratados en nuestra institución por carcinoma de células renales (CCR). Comparamos los pacientes clasificados en el estadio T1NOMO según la revisión TNM de 1997 con la clasificación según la revisión de 1992 para establecer diferencias de supervivencia. Comparamos diferentes cortes por tamaño tumoral y valoramos su implicación en la supervivencia.

MATERIAL Y MÉTODOS: Análisis retrospectivo de una serie de 168 pacientes intervenidos por CCR, de ellos 72 clasificados en estadio T1NOMO. Comparamos supervivencia según las dos últimas revisiones TNM y en tres grupos de tamaño: $1-3 \mathrm{~cm}, 3-5 \mathrm{~cm}$ y $5-7 \mathrm{~cm}$.

RESULTADOS: Hallamos menor supervivencia en los pacientes $\mathrm{T} 1^{1997}(\mathrm{p}=0,0478)$ y en grupo de tamaño 5-7 cm, con respecto al $1-3 \mathrm{~cm}(\mathrm{p}=0,02)$ y al de 3-5 $\mathrm{cm}(\mathrm{p}=0,0465)$.

CONCLUSIONES: En nuestra serie, la revisión de 1997 en el estadio T1 supone un descenso de supervivencia. El límite en $7 \mathrm{~cm}$ es excesivo, lo consideramos más apropiado por debajo de $5 \mathrm{~cm}$.

PALABRAS CLAVE: Carcinoma de células renales. Supervivencia. Estadio TNM. Tamaño tumoral.

\section{ABSTRACT}

"CUT-OFF POINT REVISION BETWEEN 1997 TNM CLASSIFICATION STAGES T1 AND T2 OF RENAL CARCINOMA"

OBJECTIVE: We retrospectively review the patients treated at our institution for renal cell carcinoma (RCC). We compare the patients classified in TNM state T1NOMO in the 1997 revision with the 1992 one in order to determine survival differences. We divide patients in three size related groups and compare its survival rates.

MATERIAL AND METHODS: We review 168 chyrurgically treated patients. 72 of them were classified into T1NOMO stage. We compare cancer-free survival in patients included in 1997 and $1992 \mathrm{~T} 1 \mathrm{stage}$. We divide patients in three groups: $1-3 \mathrm{~cm}, 3-5 \mathrm{~cm}, 5-7 \mathrm{~cm}$ and compare respective cancer-free survival.

RESULTADOS: There is a survival difference between $\mathrm{T} 1^{1997}-\mathrm{T} 2{ }^{1992}(\mathrm{p}=0.478)$. There is an inferior survival in size group 5-7 cm compared with $1-3 \mathrm{~cm}$ and $3-5 \mathrm{~cm}$ ones $(\mathrm{p}=0.02 / 0.0465)$.

CONCLUSIONS: In our patients, 1997 revision of T1 size supposes a descent of cancer-free survival compared with 1992 one. We consider a better stage limit under $5 \mathrm{~cm}$, instead of actual $7 \mathrm{~cm}$. 
$\mathrm{E}^{1}$ carcinoma de células renales (CCR) es un tumor relativamente raro y supone aproximadamente el $3 \%$ de los tumores renales malignos en el adulto ${ }^{1}$.

El primer sistema de estadiaje del CCR fue propuesto por Flocks y Kadesky en 1958 y en base a él todas las clasificaciones posteriores identificaron tres categorías pronósticas principales: enfermedad limitada al riñón, extensión local y extensión a distancia ${ }^{2}$. Clásicamente el estadio patológico fundamentado en la clasificación TNM ha sido considerado como el factor de pronóstico y supervivencia más importante ${ }^{3}$; y en los estudios multivariantes publicados hasta la fecha indican que el tamaño tumoral es el factor independiente asociado a la supervivencia más importante.

En 1997 la Unión Internationale Contre le Cancer (UICC) introdujo cambios en la clasificación TNM del CCR; considerando que el tamaño tumoral que limitaba el estadio T1 del T2 estaba en $7 \mathrm{~cm}^{4}$.

Tras esta nueva clasificación han surgido publicaciones en la literatura que consideran este límite demasiado alto y que promulgan que la supervivencia en el estadio T1NOMO ha disminuido con respecto a la clasificación TNM de 1992 ${ }^{5-7}$.

El objetivo de nuestro estudio es la revisión de nuestra experiencia en 12 años de los casos diagnosticados post-operatoriamente en el Servicio de Urología del Hospital Reina Sofía de Córdoba como carcinoma de células renales T1NOMO, según la clasificación TNM/97, realizando un análisis descriptivo de los casos y un análisis de supervivencia, intentando determinar si existen diferencias significativas con los tumores clasificados según la $\mathrm{TNM}^{92}$ y según el tamaño tumoral.

\section{MATERIAL Y MÉTODOS}

Desde enero/88 hasta diciembre/00 hemos obtenido una muestra de $168 \mathrm{CCR}, 72$ de ellos clasificados post-operatoriamente como pT1NOMO $(42,8 \%)$. Realizamos un análisis descriptivo de los casos y un análisis de supervivencia comparando la muestra en función del tamaño tumoral, considerando la clasificación TNM/92 versus la clasificación TNM/97.

Asímismo, hemos dividido arbitrariamente nuestros casos en tres grupos utilizando el tamaño tumoral como factor de clasificación: grupo I (0-3 $\mathrm{cm})$; grupo II $(3-5 \mathrm{~cm})$ y grupo III $(5-7 \mathrm{~cm})$, comparando la supervivencia entre los tres grupos.

Realizamos un análisis estadístico descriptivo y un análisis de supervivencia mediante tablas de Kaplan-Meyer, comparando supervivencias mediante el test de Log-Rank con el programa estadístico para entorno Windows (Figuras 1, 2, 3, 4 y 5).

\section{RESULTADOS}

De los 72 casos obtenidos, 42 pacientes eran hombres $(58,3 \%)$ y 30 mujeres $(41,7 \%)$. La edad media fue de 58,86 años $(S=11,96$; $R=30-84$; $\mathrm{MED}=61,5)$ y el tiempo medio de seguimiento de 49,19 meses ( $\mathrm{S}=28,22, \mathrm{R}=7-120$; $\mathrm{MED}=45,5)$.

En relación con el tamaño tumoral, 1 caso presentaba un tamaño de $1 \mathrm{~cm}, 4$ de $2 \mathrm{cms}, 10$ de 3 cms, 19 de $4 \mathrm{cms}, 17$ de $5 \mathrm{cms}, 16$ de $6 \mathrm{cms}$ y 5 de $7 \mathrm{cms}$. Con respecto a la localización tumoral, la más frecuente fue el riñón derecho en 43 casos (59,7\%); en el riñón izquierdo se localizaron 29 casos (40,3\%).

El tipo de cirugía que más frecuentemente se realizó fue la nefrectomía radical en 52 casos (75\%), seguida de la nefrectomía parcial 10 casos $(13,9 \%)$, tumorectomía 5 casos $(5,6 \%)$ y nefrectomía simple 5 casos $(5,6 \%)$. La estancia media hospitalaria fue de 10,44 días $(\mathrm{S}=5,18 ; \mathrm{R}=6-34$; $\mathrm{MED}=9$ ).

La forma de presentación más frecuente fue el hallazgo incidental 26 casos (36,1\%), seguido del dolor 17 casos (23,6\%), hematuria $12(16,7 \%)$, HTA $2(2,8 \%)$, otros $4(5,6 \%)$. No se determinó la clínica inicial en 9 pacientes $(12,5 \%)$. La asociación de dolor y hematuria se produjo en 2 casos $(2,8 \%)$.

En relación con el tumor incidental, la ecografía fue la prueba que determinó el diagnóstico inicial en 20 casos (77\%) y el estudio del S. Prostático la causa más frecuente del inicio de la batería diagnóstica en este subgrupo, 12 pacientes (46\%).

Se produjeron 8 complicaciones médicas postoperatorias $(11,1 \%)$, neumonía en 3 tres casos $(4,2 \%)$ y HDA en $3(4,2 \%)$ fueron las más frecuentes; se produjeron así mismo 9 complicaciones quirúrgicas post-operatorias (13\%), la infección de la herida $5(6,9 \%)$ y el hematoma de la herida 2 $(2,8 \%)$ fueron las más frecuentes. 11 pacientes requirieron transfusión de concentrado de hematíes intra o post-operatoriamente $(15,3 \%)$. 


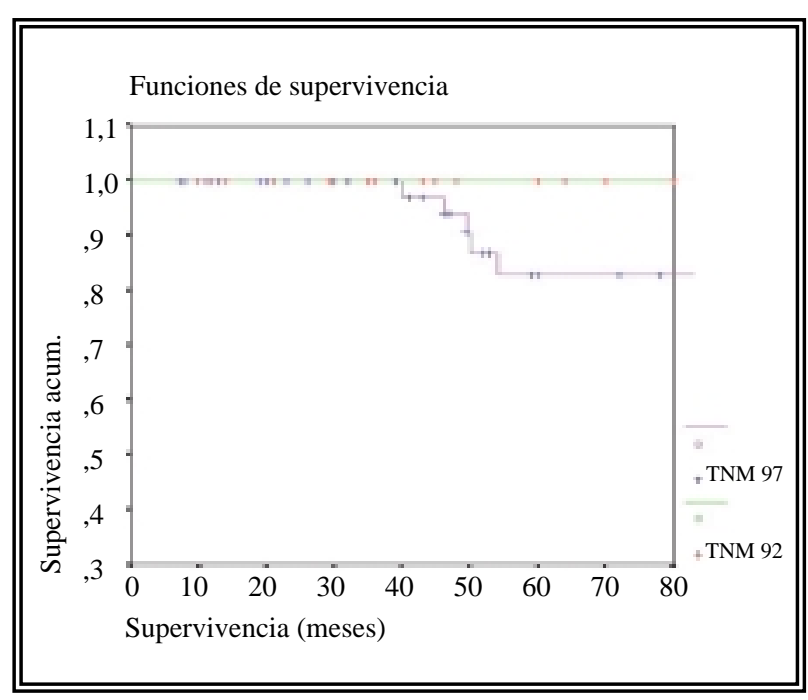

FIGURA 1. Comparando supervivencia TNM ${ }^{92}$ vS $T N M^{97}$.

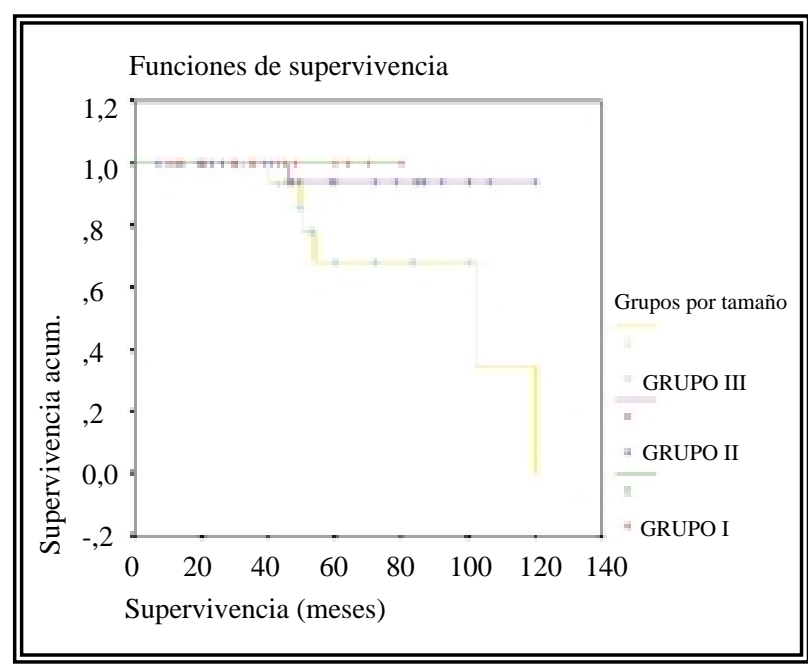

FIGURA 2. Comparando supervivencia en los tres grupos.

En el estudio global del grupo pT1NOMO, la supervivencia media fue de 49,43 meses ( $\mathrm{S}=28,19$; Med=46), a la finalización del estudio 12 pacientes han fallecido, de los cuales $8(11,1 \%)$ lo hicieron por recidiva o progresión tumoral.

Comparando el subgrupo $\mathrm{T}^{\mathrm{TNM} / 92}$ con el $\mathrm{T} 1^{\mathrm{TNM} / 97}$ la probabilidad de estar vivo a los 5 años es del 100 y $83,34 \%$ respectivamente $(\mathrm{p}=0,0478)$. Comparando los subgrupos GI (tumores con un tamaño entre 0-3 cms), GII (tumores con un tamaño entre $3-5 \mathrm{cms}$ ) y GIII (tumores con un tamaño entre 5-7 cms), la probabilidad de supervivencia a los 5 años es del 100\%, 93,45\% y 67,56\% respectivamente $(p=0,0404)$. Si comparamos grupos I y

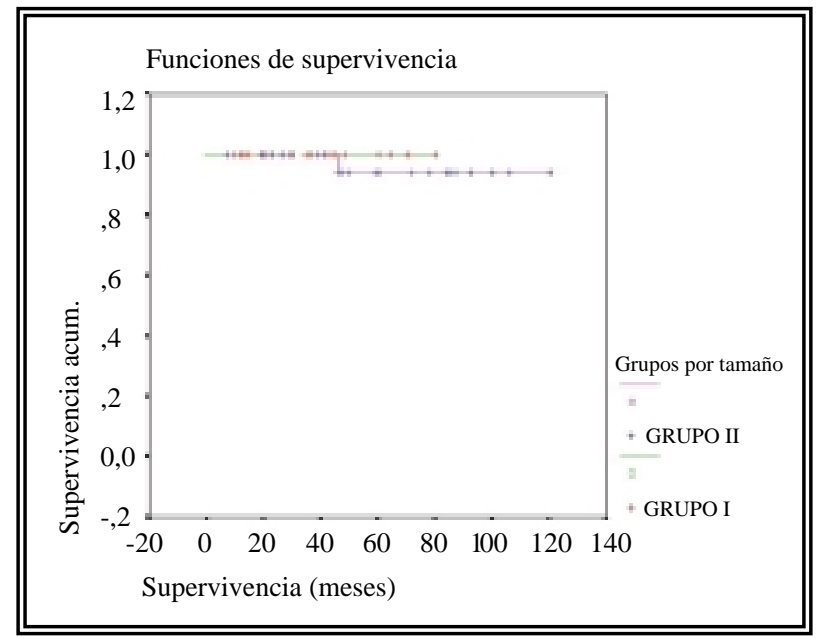

FIGURA 3. Comparando supervivencia entre grupo I y II.

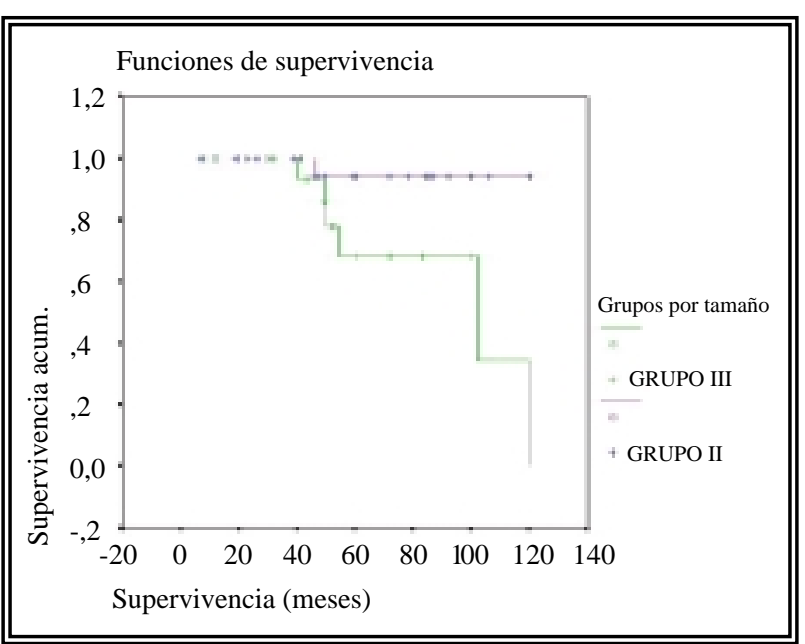

FIGURA 4. Comparando supervivencia entre grupo II y III.

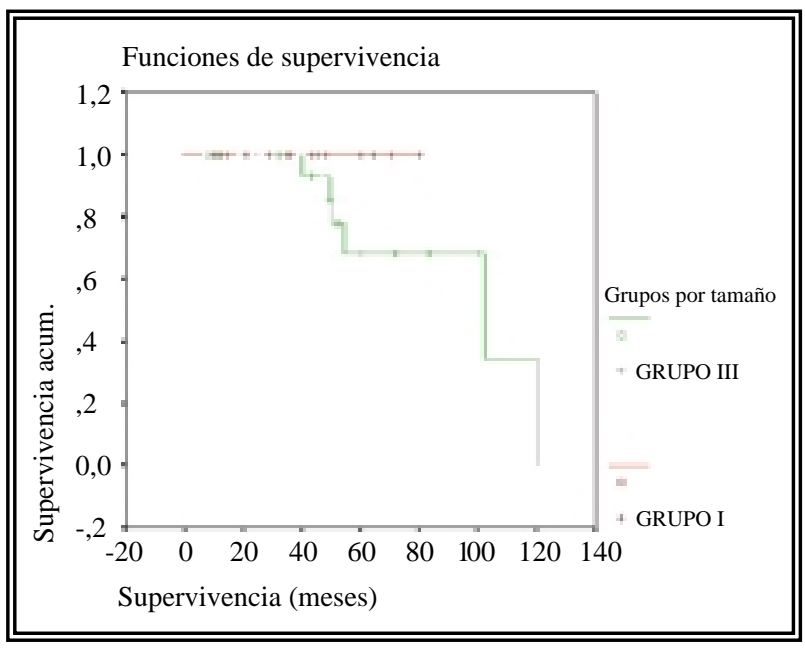

FIGURA 5. Comparando supervivencia entre grupo I y III. 
II, grupos II y III y grupos I y III, no encontramos diferencias estadísticamente significativas en relación a la supervivencia entre grupo I y II $(\mathrm{p}=0,735)$ pero sí, cuando comparamos estos grupos de forma individual con el grupo III ( $\mathrm{p}=0,02 /$ $\mathrm{p}=0,0465)$.

\section{DISCUSIÓN}

Desde que en 1938 Bell $^{8}$ sugiriera basándose en sus estudios sobre autopsias que el tamaño tumoral tiene relación con el desarrollo de metástasis en el CCR, se le ha considerado un factor pronóstico. La clasificación TNM para los dos primeros estadios (confinados al riñón) se basa en el tamaño tumoral.

En estadios precoces, el CCR debe ser considerado como un tumor maligno que debe ser abordado quirúrgicamente con intención curativa; asî mismo en las últimas dos décadas la cirugía conservadora permite, en los casos seleccionados, tasas de supervivencia similares a la cirugía radical $^{7}$.

En nuestro estudio planteamos un punto de conflicto analizando como factor de supervivencia el tamaño tumoral y concluyendo que los tumores superiores a 5 centímetros tienen una tasa de mortalidad cáncer específica mayor; así mismo, los resultados de nuestra serie indican una mayor tasa de mortalidad en el subgrupo T1NOMO de la clasificación de 1997, con respecto a este mismo grupo en la clasificación precedente. Estos datos coinciden con los publicados en la literatura reciente ${ }^{5,6}$.

Novick et al. ${ }^{7}$ en su revisión publicada en 1999 sobre la cirugía conservadora en el cáncer renal apuntaba que, según la clasificación TNM/97, existían tumores encuadrados en el estadio T1NOMO que no podían ser considerados para técnicas de nefrectomía parcial pues las tasas de recidiva y progresión tumoral eran mayores. Previamente a la revisión TNM de 1997, series amplias de pacientes tratados mediante cirugía conservadora ${ }^{9}$ sugerían un seguimiento diferente atendiendo al estadio pT1 y pT2, limitado en 2,5 $\mathrm{cm}$. Zismann et al. ${ }^{5}$, en una publicación reciente, establece el punto de corte en $4,5 \mathrm{cms}$ y aboga por una subclasificación del estadio T1 en Tla y T1b, estableciendo como límite este tamaño. Igarashi et al. ${ }^{6}$ establecen en su serie el punto de corte en 4 centímetros, evaluando otros factores que pudieran influir en la supervivencia como el grado tumoral, la microinvasión vascular, la localización del tumor, la edad del paciente y la histopatología tumoral. Sin embargo, Nativ et al. ${ }^{10}$ no encuentran diferencias en cuanto al tamaño tumoral y sugieren que la diferencia puede estribar en un menor tiempo de evolución de los tumores más pequeños, que aún no les ha permitido desarrollar metástasis.

El carecer de un análisis multivariado, de un mayor número de casos y de un tiempo de seguimiento mayor son puntos criticables en nuestro estudio. Otro punto débil de nuestro estudio supone no haber tenido en cuenta otros factores que influyen en la supervivencia y que han sido considerados en series recientes, además de la clasificación TNM y el tamaño tumoral, para inferir un pronóstico de la enfermedad, un estudio interesante al respecto publicado por Kattan et al. ${ }^{11}$ establece un nomograma en el que evalúan postoperatoriamente el tamaño tumoral, tipo histológico, clasificación $\mathrm{TNM}^{97}$ y sintomatología, con la que se obtiene una puntuación que permite inferir la probabilidad de supervivencia a 5 años. Sin embargo, el propósito de esta revisión se ha centrado exclusivamente en determinar la influencia del tamaño en la supervivencia y sus implicaciones en una posible modificación de la clasificación TNM.

La futura aplicación clínica de marcadores genéticos tumorales de implicación pronóstica nos permitirán un mejor conocimiento y abordaje de esta patología ${ }^{12-14}$ y un estadiaje tumoral relacionado con las características pronósticas reales de las lesiones.

Recientemente la UICC ha revisado el estadio T1 para el carcinoma de células renales estableciendo dos subgrupos: el subgrupo Tla para neoplasias menores de 4 centímetros y el Tlb para neoplasias entre 4-7 centímetros, que será de obligada referencia a partir del 1 de enero de $2003^{15}$.

\section{CONCLUSIONES}

Atendiendo exclusivamente al tamaño tumoral se hace necesaria una modificación de la clasificación en los dos primeros estadios TNM, que tienen en cuenta sólo este factor para establecer el límite ya que los datos obtenidos en nuestra serie 
sugieren una diferencia en la supervivencia entre los tamaños mayores de cinco centímetros y los menores de este diámetro. Sugerimos diferenciar en cuanto a pronóstico los tumores menores de cinco centímetros con respecto a los mayores.

\section{REFERENCIAS}

1. WALSH, RETIK, VAUGHAN, WEIN.: Campbell's Urology. Seventh edition; 2: 2293.

2. REQUENA MJ, LÓPEZ BELTRÁN A, ANGLADA F.J.: Neoplasias renales. Diagnóstico y tratamiento. Pág. 76. I.S.B.N. 84-606-3125-7.

3. JAVIDAN J, STRICKER HJ, TAMBOLI P, AMIN MB, PEABODY JO, DESHPANDE A, MENON M, AMIN MB.: Prognostic significance of the 1997 TNM classification of renal cell carcinoma. The Journal of Urology 1999; 162: 1277.

4. GUINAN P, SOBIN L, ALGABA F et al.: TNM staging of renal cell carcinoma: Workgroup № 3 . Union Internationale contre le cancer and the American Joint committee on cancer. Cancer 1997, 80: 992.

5. ZISMAN A, PANTUCK AJ, CHAO D, DOREY F, SAID JW, GITLITZ BJ, DEKERNION JB, FIGLIN RA, BELLDEGRUN AS.: Reevaluation of the 1997 TNM classification for renal cell carcinoma: $\mathrm{T} 1$ and $\mathrm{T} 2$ cut-off point at 4.5 rather than $7 \mathrm{~cm}$. Better correlates with clinical outcome. The Journal of Urology 2001; 166: 54-58.

6. IGARASHI T, TOBE T, NAKATSU HO, SUZUKI N, MURAKAMI S, HAMANO M, MARUOKA M, NAGAYAMA T, MATSUZAKI O, ITO H.: The impact of a $4 \mathrm{~cm}$ cut-off point for stratification of T1NOMO renal cell carcinoma after radical nephrectomy. The Journal of Urology 2001; 165: 1103-1106.

7. UZZO RG, NOVICK AC.: Nephron sparing surgery for renal tumors: indications, techniques and outcomes. J Urol 2001 jul; 166: 6-18.

8. BELL ET.: A classification of renal tumours with observation on the frequency of various types. J Urol 1938; 39: 238.
9. HAFEZ KS, NOVICK AC, STEVEN C.: Campbell. Patterns of tumor recurrence and guidelines for followup after nephron sparing surgery for sporadic renal cell carcinoma. J Urol 1997 jun; 157: 20672070.

10. NATIV O, SABO E., RAVIV GIL, MADJAR S, HALACHMI S, MOSKOVITZ B.: The impact of tumor size on clinical outcome in patients with localized renal cell carcinoma treated by radical nephrectomy. J Urol 1997 sept; 158: 729-732.

11. KATTAN M, REUTER V, MOTZER R, KATZ J, RUSSO P.: A postoperative prognostic nomogram for renal cell carcinoma. J Urol 2001 jul; 166: 63-67.

12. HOFMOCKEL G, TSATALMAS P, MULLER H, DAMMRICH J, POOT M, MAURER-SCHULTZE B, MULLER-HERMELINK HK, FROHMULLER HG, BASSUKAS ID.: Significance of conventional and new prognostic factors for locally confined renal cell carcinoma. Cancer 1995 jul 15; 76 (2): 296-306.

13. GELB AB, SUDILOVSKY D, WU CD, WEISS LM, MEDEIROS LJ.: Appraisal of intratumoral microvessel density, MIB-1 score, DNA content, and p53 protein expression as pronogstic indicators in patients whith locally confined renal cell carcinoma. Cancer 1997 nov 1; 80 (9): 1768-1775.

14. SLATON JW, INOUE K, PERROTTE P, EL-NAGGAR AK, SWANSON DA, FIDLER IJ.: Expression levels of genes that regulate metastasis and angiogenesis correlate with advanced pathological stage of renal cell carcinoma. Am J Pathol 2001 feb; 158 (2): 735-4.

15. TNM classification of malignant tumours $6 / \mathrm{E}$ june 2002.

Dr. A.J. López Luque

Pasaje Santa Rosalía, 4 - 2o 4

14010 Córdoba

(Trabajo recibido el 2 diciembre de 2002) 